\title{
Evolution des caractères spermatiques avec l'âge
}

\author{
Louis SIBERT1, Nathalie RIVES2 \\ 1 Service d'Urologie, \\ 2 Laboratoire de Cytologie, Cytogénétique et Biologie de La Reproduction, CHU de Rouen
}

\begin{abstract}
RESUME
L'allongement de l'espérance de vie, l'augmentation de l'âge parental du fait des conditions socio-économiques dans les pays développés, conjointement aux progrès des techniques d'assistance médicale à la procréation expliquent l'intérêt récent porté sur la fertilité des hommes âgés. Les modifications hormonales (déficit testiculaire primaire, diminution de l'amplitude des pics de sécrétion hypothalamique de GnRH), les altérations histologiques et morphologiques testiculaires (sclérose artériolaire, dégénérescence des cellules de Leydig et de Sertoli, raréfaction des cellules germinales, épaississement de l'albuginée testiculaire) en rapport avec le vieillissement physiologique sont relativement bien documentées. Le retentissement de ces modifications physiologiques sur la qualité spermatique du sujet âgé est peu aisé à établir de façon nette, en raison du peu de données disponibles et de la grande variabilité interindividuelle des paramètres spermatiques. Globalement, la qualité spermatique diminue progressivement avec l'âge, sans qu'un âge seuil puisse être individualisé. Les altérations observées portent surtout sur le volume de l'éjaculât, la mobilité et la morphologie des spermatozoïdes. La numération semble moins affectée. Cependant, tous les autres facteurs pathologiques et psychosociaux fréquemment observés chez les sujets âgés, doivent être pris en compte dans l'interprétation des données (modifications de l'appareil vésico-sphinctérien et du tractus génital, affections chroniques, prises médicamenteuses, tabagisme, diminution de la fréquence des rapports sexuels, âge de la partenaire). Des études comparatives supplémentaires, incluant un plus grand nombre de patients âgés avec des tranches d'âge clairement individualisées, avec des populations appariées pour les autres facteurs d'altération de la qualité spermatique, sont nécessaires. Au total, l'analyse de la littérature ne permet pas d'individualiser des critères pronostiques spécifiques de l'éventuelle fécondité en rapport avec l'âge. Quelles que solent les caractéristiques spermatiques d'un sujet âgé désireux d'une éventuelle paternité, ceuxci ne permettent pas de faire l'économie d'un bilan clinique et andrologique complet.
\end{abstract}

Mots clés : âge, fertilité, volume spermatique, numération, mobilité, morphologie

\section{INTRODUCTION}

Alors que l'effet du vieillissement sur la fertilité féminine est bien documenté, les fonctions de reproduction chez l'homme âgé sont moins bien connues. Le peu de données disponibles peut être expliqué par l'absence, jusqu'à un passé récent, d'incidence pratique des conséquences du vieillissement sur la fertilité masculine. En effet, il était communément admis que la majorité des hommes d'un certain âge n'étaient pas ou plus demandeurs d'une paternité.

L'amélioration de l'espérance de vie est particulièrement nette dans les pays développés. La conséquence en est un vieillissement de la population. L'augmentation de l'âge parental lié aux conditions socio-économiques et les progrès récents des techniques d'assistance médicale à la procréation sont les principaux artisans de l'attention nouvelle portée sur la fertilite potentielle des hommes âgés. L'étude des modifications physiopathologiques des fonctions de reproduction masculine liées au vieillissement prend ainsi un intérêt croissant. L'analyse des paramètres spermatiques est généralement considérée comme un marqueur fiable de la fertilité masculine.

Dans la mesure où les considérations pronostiques et thérapeutiques concernant la fertilité des patients en découlent directement, il paraît intéressant de faire le point sur les données actuellement disponibles concernant la qualité spermatique chez les sujets âgés.

\section{Correspondance :}

Dr Louis SIBERT - Service d'Urologie, CHU de Rouen, Hôpital Charles-Nicolle, 1 rue de Germont, 76031 Rouen Cedex. France - Tel 02.32.88.81.73 - Fax 02.32.88.82.05 - Email :louis.sibert@chu-rouen.fr 


\section{VARIATIONS PHYSIOLOGIQUES LIEES A L'AGE}

S'il faut reconnaître que les conséquences exactes du vieillissement physiologique sur la fertilité masculine sont difficiles à établir, des études histologiques et endocrinologiques de plus en plus précises ont permis d'identifier des altérations progressives des fonctions endocrine et exocrine testiculaires.

\section{Modifications hormonales liées au vieillissement}

\section{a) Fonction testiculaire endocrine}

En dehors de tout contexte morbide ou pathologique, la sécrétion de testostérone décroît avec l'âge. Le taux de testostérone plasmatique se réduit progressivement après 45 ans $[3,8,22,23]$. La décroissance spontanée de la testostérone plasmatique est cependant masquée par une élévation progressive du taux de TeBG (Testosterone Binding Globulin). Ces deux facteurs conjugués expliquent la diminution constante de la fraction libre du stéroïde qui se réduit de plus de $50 \%$ entre 50 et 90 ans. Les facteurs responsables de cette modification sont multiples :

a) La réduction de la capacité stéroïdogène du testicule, qui se manifeste initialement par une perte du rythme circadien de la testostérone, est objectivée par la réduction de la réponse testiculaire à l'administration d'une dose pharmacologique d'hCG [3]. Ce phénomène a été rattaché à une réduction du nombre de cellules de Leydig [15]. Parallèlement, la déflation de l'activité des tubes séminifères se traduit par une diminution de la capacité de rétrocontrôle négatif sur la sécrétion hypophysaire de $\mathrm{FSH}$. Ce phénomène de déficit testiculaire primaire est néanmoins inconstant et n'est globalement observé que chez $50 \%$ des hommes âgés.

b) L'altération du fonctionnement hypothalamo-hypophysaire de I'homme âgé : si la rythmicité des pulses de $\mathrm{GnRH}$ reste stable, leur amplitude se réduit progressivement avec l'âge. Ceci a été rattaché à une modification de la sensibilité hypothalamique au rétrocontrôle stéroïdien $[3,22]$.

Les responsables des modifications physiologiques liées à l'âge apparaissent donc à la fois testiculaire (déficit primaire) et hypothalamique (déficit sécrétoire en $\mathrm{GnRH}$ ). De plus, le système de réception aux androgènes semble également modifié. La concentration intracellulaire des récepteurs aux androgènes se réduit avec l'âge, diminuant d'autant la concentration intra tissulaire en testostérone et consécutivement les effets androgéniques sur les voies spermatiques intra et extratesticulaires.

\section{b) Fonction testiculaire exocrine}

La fonction testiculaire exocrine est également modifiée au cours du vieillissement. L'inhibine B plasmatique est une glycoprotéine sécrétée par les cellules de Sertoli sous l'influence de la FSH. Elle exerce un effet de rétrocontrôle sur la sécrétion de FSH. L'augmentation de la concentration plasmatique de $\mathrm{FSH}$ et la diminution de la concentration circulante d'inhibine $B$ chez les sujets âgés rendent compte des altérations fonctionnelles des tubes séminifères [8, 18].

\section{Modifications histo-morphologiques liées au vieillis- sement}

L'influence du vieillissement sur l'histologie testiculaire a fait l'objet d'un certain nombre de travaux, mais jusqu'à présent cette évolution n'a pas été observée jusqu'à un âge très avancé $[2,8]$. Un des premiers signes décrit est la sclérose artériolaire provoquant une ischémie progressive du tissu testiculaire. A partir de $35-40$ ans, la densité des capillaires entourant les tubes séminifères diminue régulièrement avec l'âge. Des signes de dégénérescence des cellules de Leydig, une réduction de leur nombre sont observés parallèlement à la baisse de la testostérone plasmatique [15]. Au niveau des tubes séminifères, plusieurs modifications ont été décrites : un épaississement de la membrane basale des tubes, une réduction de leur diamètre, des anomalies des cellules de Sertoli et une diminution progressive du nombre de cellules germinales commençant par les spermatogonies puis les spermatozoïdes avec un arrêt fréquent au stade pachytène [2, 13]. Les altérations sertoliennes et germinales se produisent simultanément sans que l'on puisse déterminer quelle est la cause et quelle est la conséquence [2].

On sait également que des modifications enzymatiques aboutissent à une diminution d'efficacité de la barrière hémato-testiculaire et que le vieillissement des cellules de Sertoli aboutit à une chute de la production d'ABP (Androgen Binding Protein). Des études font mention d'une diminution de l'épaisseur de l'albuginée dans environ $30 \%$ des cas. Une réduction du volume testiculaire est également observée, bien qu'elle ne semble pas être significative entre les tranches $21-50$ ans et $51-80$ ans $[12,18]$.

Une des caractéristiques principales du vieillissement testiculaire est la coexistence d'altérations majeures dans certains tubes séminifères alors que d'autres tubes ont un aspect normal. La grande variation interindividuelle est une autre constante des anomalies histologiques du vieillissement testiculaire.

\section{CONSEQUENCES SUR LES PARAMETRES SPERMATIQUES}

\section{Le volume spermatique (Tableau 1)}

Dans une étude portant sur plus de 800 patients, le volume spermatique ne semble pas modifié par l'âge ; malheureusement aucun patient âgé de plus de 50 ans n'était inclus dans cette étude [20]. Dans leur méta-analyse regroupant les résultats de plus de 20 études publiées entre 1980 et 1999, Kidd et al. [11] retrouvent une diminution du volume de l'éjaculât oscillant de 3 à $22 \%$ chez les patients âgés de plus de 50 ans, comparativement à des patients de moins de 50 ans, mais seulement cinq études avaient pris en compte le délai d'abstinence. Plus récemment, Jung et al. [9], retrouvent une diminution du volume spermatique de $29 \%$ entre un groupe de 134 jeunes patients ( 24 ans en moyenne) et un groupe de 66 patients de plus de 50 ans. 


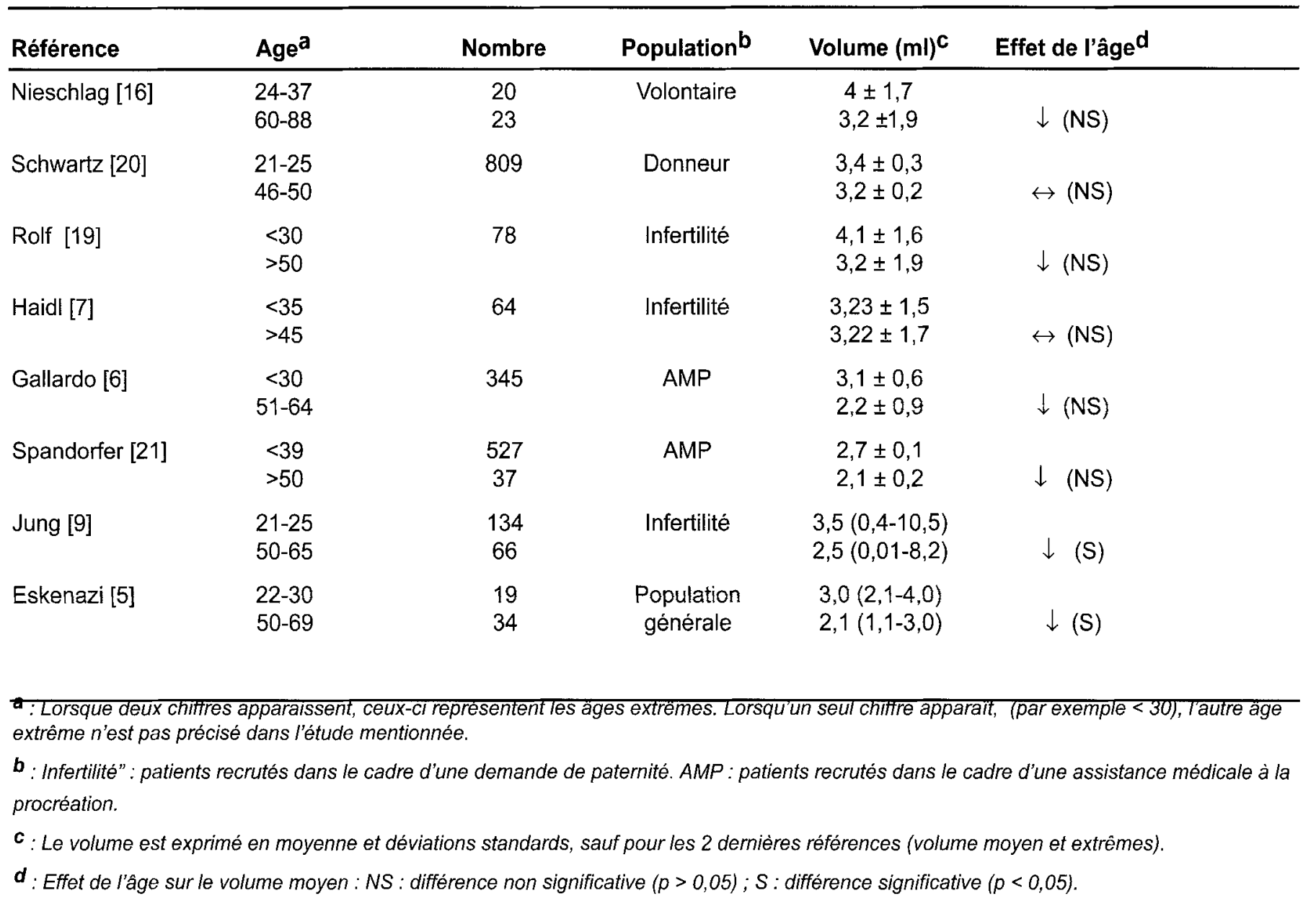

Sur une population de 97 hommes sains âgés de 22 à 80 ans et après ajustement par le délai d'abstinence, les antécédents de consommation d'alcool et de tabac, Eskenasi et al. [5] ont retrouvé une diminution du volume de $0,03 \mathrm{ml}$ par année. Leurs patients de 50 ans ont une diminution relative du volume spermatique de $20 \%$ par rapport aux sujets de 30 ans. Les analyses de régression linéaire estiment qu'à 30 ans, $10 \%$ des hommes sont susceptibles d'avoir un volume spermatique faible (> $2 \mathrm{ml}$ ) et que cette proportion serait de $35 \%$ parmi les hommes de 50 ans et de $80 \%$ parmi les hommes de 80 ans.

\section{La numération}

Dans une étude comparative des fonctions testiculaires de jeunes pères (24-37 ans) et de patients âgés (60-88 ans), Nieschlag et al. [16] rapportent une élévation de la numération avec l'âge. Au contraire, Haidl et al. [7] n'ont pas retrouvé de différence entre un groupe de pères jeunes $(32,2$ ans de moyenne) et un groupe de pères plus âgés $\langle 50,3$ ans de moyenne). Auger et al. [1] retrouvent une diminution de la concentration spermatique de $3,3 \%$ par année d'âge, et une diminution de la concentration des hommes de 50 ans de l'ordre de $66 \%$ par rapport aux hommes de 30 ans. II faut préciser que les données de cette étude ont été recueillies sur un large échantillon (plus de
1000 donneurs de 19 à 59 ans), incluant un groupe témoin et tenant compte de la durée d'abstinence et d'autres facteurs pathologiques. Jung et al. [9] ne retrouvent pas de différence significative de numération ou de concentration entre les patients âgés de 21 à 25 ans et les sujets âgés de 50 à 65 ans (Tableau 2). Par contre, il semble y avoir un plus grand nombre de patients avec une numération totale anormale dans les tranches d'âge les plus élevées. Ainsi la probabilité d'avoir une numération totale anormale serait de $5 \%$ à l'âge de 30 ans, de $10 \%$ à 50 ans et de $35 \%$ à 80 ans. Le nombre de patients affectés par tranche d'âge reste cependant faible et les différences globales peu importantes. Du fait de la diminution du volume, l'étude de la numération totale de spermatozoïdes par éjaculât serait un critère de mesure plus fiable que la concentration (nombre de spermatozoïdes par $\mathrm{ml}$ ).

\section{La mobilité}

D'après la méta-analyse de Kidd [11], 19 études ont mesuré spécifiquement la mobilité des spermatozoïdes en fonction de l'âge entre 1980 et 1999 , le plus souvent par microscopie optique. La majorité des études ayant une méthodologie correcte retrouve constamment une nette diminution de la mobilité avec l'âge, variant de 3 à $37 \%$ entre les patients de 30 ans et ceux de 50 ans [1, 9, 11]. L'altération 
Tableau 2 : Evolution de la concentration et de la numération totale de spermatozoïdes avec l'âge.

\begin{tabular}{|c|c|c|c|c|c|}
\hline Référence & $\mathrm{Age}^{\mathrm{a}}$ & Nombre & Population $\mathbf{b}$ & Concentration ${ }^{\mathrm{C}}\left(10^{6} / \mathrm{ml}\right)$ & Numération totale $\left(10^{6}\right)$ \\
\hline Nieschlag [16] & $\begin{array}{l}24-37 \\
60-88\end{array}$ & $\begin{array}{l}20 \\
23\end{array}$ & Volontaire & $\begin{array}{c}78 \pm 51 \\
120 \pm 101\end{array}$ & n.d. \\
\hline Schwartz [20] & $\begin{array}{l}21-25 \\
46-50\end{array}$ & 809 & Donneur & $\begin{array}{l}97 \pm 13,1 \\
105 \pm 14\end{array}$ & n.d. \\
\hline Rolf [19] & $\begin{array}{l}<30 \\
>50\end{array}$ & 78 & Infertilité & $\begin{array}{l}44 \pm 55,3 \\
56 \pm 90,1\end{array}$ & n.d. \\
\hline Haidl [7] & $\begin{array}{l}<35 \\
>45\end{array}$ & 64 & Infertilité & $\begin{array}{l}115,1 \pm 103 \\
66,9 \pm 66,6\end{array}$ & n.d. \\
\hline Spandorfer [21] & $\begin{array}{l}<39 \\
>50\end{array}$ & $\begin{array}{c}527 \\
37\end{array}$ & AMP & $\begin{array}{l}22,7 \pm 1,3 \\
19,8 \pm 4,5\end{array}$ & n.d. \\
\hline Jung [9] & $\begin{array}{l}21-25 \\
50-65\end{array}$ & $\begin{array}{c}134 \\
66\end{array}$ & Infertilité & $\begin{array}{l}29(0-320) \\
29(0-512)\end{array}$ & $\begin{array}{l}86(0-1550) \\
73(0-1187)\end{array}$ \\
\hline Eskenazi [5] & $\begin{array}{l}22-30 \\
50-69\end{array}$ & $\begin{array}{l}19 \\
34\end{array}$ & Population générale & $\begin{array}{c}92(52-177) \\
101(42-170)\end{array}$ & $\begin{array}{l}345(109-658) \\
235(55-297)\end{array}$ \\
\hline
\end{tabular}

a : Lorsque deux chiffres apparaissent, ceux-ci représentent les âges extrêmes. Lorsqu'un seul chiffre apparait, (par exemple < 30), l'autre âge extrême n'est pas précisé dans l'étude mentionnée

b : Infertilité" : patients recrutés dans le cadre d'une demande de patemité. AMP : patients recrutés dans le cadre d'une assistance médicale à la procréation.

$c$ : La concentration est exprimée en moyenne et déviations standards, sauf pour les 2 dernières références (concentration moyenne et extrêmes).

n.d. : non déterminée.

de la mobilité avec l'âge touche le pourcentage de formes normo-mobiles (spermatozoïdes flêchants) et le nombre total de spermatozoïdes mobiles. D'après Eskenazi et al. [5], le pourcentage de formes mobiles diminue de $0,7 \%$ par année et le nombre total de formes mobiles diminue de $4,7 \%$ par an. Un patient de 50 ans a un pourcentage de formes mobiles diminué de $28 \%$ par rapport à celui d'un homme de 30 ans. Le nombre de patients ayant une diminution du pourcentage de formes mobiles augmente significativement par tranche d'âge. Ces données sont résumées sur le Tableau 3.

\section{La morphologie (Tableau 4)}

Neuf études sur les 14 ayant examiné la relation entre la morphologie spermatique et l'âge, colligées par Kidd [11], confirment la corrélation entre l'âge et la diminution du pourcentage de formes normales $[1,6,7,14,19,20]$. Auger et al. [1] notamment, retrouvent une augmentation de la tératospermie de 0,9\% par année d'âge, après appariement avec l'année de naissance et la durée d'abstinence. Si on prend en compte les données des publications les plus fiables sur le plan méthodologique, la diminution du pourcentage de formes mobiles varie de 4 à $18 \%$ chez les hommes de 50 ans et plus par rapport aux patients de 30 ans et moins. L'étude récente de Jung [9] confirme ces résultats, avec une diminution encore plus marquée du pourcentage de formes normales chez les sujets âgés, de l'ordre de $44 \%$. Ce chiffre extrême est probablement expliqué par le plus grand nombre de sujets âgés (> 60 ans) inclus dans leur travail. II est à noter que la comparaison qualitative des anomalies morphologiques publiées est difficile du fait de la grande variabilité des critères de classification employés (OMS, David, critères de Dusseldorf). Cependant, la grande majorité des données disponibles confirme que la tératospermie observée, quelle que soit la classification employée, est en rapport avec des anomalies flagellaires, notamment l'enroulement des flagelles $[7,9$, $14,20]$. JUNG et al. [9], avec la classification de l'OMS, retrouvent une augmentation de $27 \%$ de la tératospermie dans leur groupe de sujets âgés, celle-ci portant essentiellement sur les flagelles (dont $59 \%$ de flagelles enroulées). Les anomalies flagellaires sont retrouvées en plus grande quantité après 50 ans dans la majorité des études [14, 20].

\section{FACTEURS PATHOLOGIQUES POUVANT MODIFIER LES PARAMETRES SPERMATIQUES LORS DU VIEILLISSEMENT}

Un certain nombre d'éléments pathologiques peuvent influencer la qualité du sperme chez l'homme âgé et doivent être pris en compte dans l'interprétation des données. Tout d'abord, de multiples causes d'origines environnementales, psychosociales ou socio-économiques, bien souvent intriquées, sont susceptibles d'altérer, avec le vieillissement, la fertilité de l'homme âgé : la diminution de la libido et de l'activité sexuelle, la plus grande fréquence des dysérections, la diminution avec l'âge de la fécondité de la partenaire. La diminution de fréquence des éjacula- 
Tableau 3 : Evolution de la mobilité spermatique avec l'âge.

\begin{tabular}{|c|c|c|c|c|c|}
\hline Référence & $\mathrm{Age}^{\mathrm{a}}$ & Nombre & Population $\mathbf{b}$ & MobilitéC (\%) & Effet de l'âge ${ }^{d}$ \\
\hline Nieschlag [16] & $\begin{array}{l}24-37 \\
60-88\end{array}$ & $\begin{array}{l}20 \\
23\end{array}$ & Volontaire & $\begin{array}{l}68 \pm 14 \\
50 \pm 19\end{array}$ & $\downarrow(S)$ \\
\hline Schwartz [20] & $\begin{array}{l}21-25 \\
46-50\end{array}$ & 809 & Donneur & $\begin{array}{l}68,9 \pm 1,8 \\
62,3 \pm 2,7\end{array}$ & $\downarrow(S)$ \\
\hline Rolf [19] & $\begin{array}{l}<30 \\
>50\end{array}$ & 78 & Infertilité & $\begin{array}{l}46 \pm 20 \\
26 \pm 22\end{array}$ & $\downarrow(S)$ \\
\hline Haidl [7] & $\begin{array}{l}<35 \\
>45\end{array}$ & 64 & Infertilité & $\begin{array}{l}30,4 \pm 14,5 \\
23,1 \pm 13,7\end{array}$ & $\downarrow$ (NS) \\
\hline Spandorfer [21] & $\begin{array}{l}<39 \\
>50\end{array}$ & $\begin{array}{c}527 \\
37\end{array}$ & AMP & $\begin{array}{l}36,5 \pm 0,9 \\
34,9 \pm 4,3\end{array}$ & $\downarrow(N S)$ \\
\hline Jung [9] & $\begin{array}{l}21-25 \\
50-65\end{array}$ & $\begin{array}{l}111 \\
56\end{array}$ & Infertilité & $\begin{array}{r}47(0-78) \\
32(0-68)\end{array}$ & $\downarrow(\mathrm{S})$ \\
\hline Eskenazi [5] & $\begin{array}{l}22-30 \\
50-69\end{array}$ & $\begin{array}{l}19 \\
34\end{array}$ & Population générale & $\begin{array}{l}50(35-60) \\
30(10-48)\end{array}$ & $\downarrow(S)$ \\
\hline
\end{tabular}

a : Lorsque deux chiffres apparaissent, ceux-ci représentent les âges extrêmes. Lorsqu'un seul chiffre apparait, (par exemple < 30), l'autre âge extrême n'est pas précisé dans l'étude mentionnée

b : Infertilité" : patients recrutés dans le cadre d'une demande de patemité. AMP : patients recrutés dans le cadre d'une assistance médicale à la procréation.

$c$ : La mobilité est exprimée en moyenne et déviations standards, sauf pour les 2 dernières références (mobilité moyenne et extrêmes).

$\boldsymbol{d}$ : Effet de l'âge sur la mobilité moyenne des spermatozoïdes : NS : différence non significative $(p>0,05) ; s$ : différence significative $(p<0,05)$.

Tableau 4 : Evolution de la morphologie des spermatozoïdes avec l'âge.

\begin{tabular}{|c|c|c|c|c|c|}
\hline Référence & $\mathrm{Age}^{\mathrm{a}}$ & Nombre & Population ${ }^{\mathbf{b}}$ & $\begin{array}{c}\% \text { de formes } \\
\text { normales }\end{array}$ & Effet de l'âge ${ }^{d}$ \\
\hline Schwartz [20] & $\begin{array}{l}21-25 \\
46-50\end{array}$ & 809 & Donneur & $\begin{array}{l}54,6 \pm 2,4 \\
54,9 \pm 1,6\end{array}$ & $\downarrow(\mathrm{S})$ \\
\hline Mladenovic [14] & $\begin{array}{l}<40 \\
>40\end{array}$ & 77 & Infertilité & $\begin{array}{l}78,5 \pm 2,9 \\
72,3 \pm 1,3\end{array}$ & $\downarrow(\mathrm{S})$ \\
\hline Rolf [19] & $\begin{array}{l}<30 \\
>50\end{array}$ & 78 & Infertilité & $\begin{array}{l}29 \pm 16 \\
26 \pm 18\end{array}$ & $\leftrightarrow(\mathrm{NS})$ \\
\hline Gallardo [6] & $\begin{array}{c}<30 \\
51-64\end{array}$ & 345 & AMP & $\begin{array}{l}61,3 \pm 32,8 \\
53,2 \pm 20,2\end{array}$ & $\downarrow(\mathrm{S})$ \\
\hline \multirow[t]{2}{*}{ Haid! [7] } & $<35$ & 64 & Infertilité & $26,5 \pm 13,9$ & \\
\hline & $>45$ & & & $20,7 \pm 16,2$ & $\downarrow$ (NS) \\
\hline \multirow[t]{2}{*}{ Spandorfer [21] } & $<39$ & 527 & AMP & $36,5 \pm 0,9$ & \\
\hline & $>50$ & 37 & & $34,9 \pm 4,3$ & $\leftrightarrow(\mathrm{NS})$ \\
\hline Jung [9] & $\begin{array}{l}21-25 \\
50-65\end{array}$ & $\begin{array}{l}111 \\
56\end{array}$ & Infertilité & $\begin{array}{c}26,5(0-70) \\
16(0-67)\end{array}$ & $\downarrow(S)$ \\
\hline
\end{tabular}

a : Lorsque deux chiffres apparaissent, ceux-ci représentent les âges extrêmes. Lorsqu'un seul chiffre apparait, (par exemple < 30), l'autre âge extrême n'est pas précisé dans l'étude mentionnée

b : Infertilité" : patients recrutés dans le cadre d'une demande de paternité. AMP : patients recrutés dans le cadre d'une assistance médicale à la procréation.

c: Le pourcentage de forme normale est exprimé en moyenne et déviations standards, sauf pour les 2 dernières références (volume moyen et extrêmes).

$\boldsymbol{d}$ : Effet de l'âge sur le pourcentage de forme normale moyen: NS : différence non significative $(p>0,05)$; $S$ : différence significative ( $p<0,05)$. 
tions pourrait masquer en partie le déclin de la spermatogenèse. Le stress, les états dépressifs influencent également la fréquence des rapports. Les prises médicamenteuses fréquentes dans la population âgée (psychotropes, diurétiques thiazidiques, anti-hypertenseurs, anti-inflammatoires non stéroïdiens), les antécédents d'infections répétées du bas appareil urinaire, de tabagisme, de consommation d'alcool, d'affections organiques chroniques (obésité, diabète, athérosclérose) influencent négativement la sexualité et la qualité spermatique $[5,10,22]$.

Certaines modifications anatomiques liées au vieillissement influencent directement la qualité spermatique. Par exemple, la diminution du volume de l'éjaculât peut probablement être reliée en grande partie, à la diminution des sécrétions des vésicules séminales. JUNG et al. [9] retrouvent une diminution significative du taux de fructose dans le sperme concomitante de la diminution du volume chez leurs patients âgés de plus de 50 ans. Les modifications physiologiques du tissu prostatique, telles que l'atrophie de la musculature lisse, les modifications de composition des sécrétions des glandes prostatiques (diminution du taux de protéine et d' $\mathrm{H}_{2} \mathrm{O}$ ) altèrent certainement le volume de l'éjaculât et la mobilité spermatique [8, 13]. Par ailleurs, le tissu épididymaire qui est hormono-sensible, joue un rôle prépondérant dans la maturation des spermatozoïdes. Le vieillissement de l'épithélium épididymaire joue un rôle non négligeable sur la diminution de la mobilité et les altérations morphologiques des spermatozoïdes $[9,10,13]$.

\section{LIMITES METHODOLOGIQUES}

La méthodologie employée par la majorité des études a certainement un impact important sur la collecte des données et doit rendre prudente l'interprétation des résultats publiés. Tout d'abord, un grand nombre d'études ont des effectifs de patients suffisamment âgés (plus de 50 ans) trop faibles (en règle, moins de 40 patients) réduisant ainsi la puissance statistique des analyses et des résultats, plus spécifiquement lorsque ces études ne rapportent pas de modification des paramètres spermatiques avec l'âge (faux négatifs) $[4,6,7,11,16,17,19,21]$. Les données proviennent généralement de comparaisons de données quantitatives entre des tranches variées et hétérogènes. Les groupes de sujets réellement âgés (plus de 70 ans) sont difficilement identifiables dans la plupart des séries. Les sujets sont généralement sélectionnés par l'intermédiaire du recrutement de centres spécialisés en infertilité et sont issus de couples pris en charge dans ces centres. Cette notion limite dans une certaine mesure la représentativité des résultats obtenus dans cette population par rapport à la population générale de patients sexuellement actifs du même âge et en bonne santé (ou fertiles ?) [5]. II faut également préciser que la plupart des facteurs médicaux et psycho-sociaux pouvant modifier la qualité spermatique au cours du vieillissement sont rarement pris en compte pour l'interprétation des paramètres spermatiques. Les patients inclus sont trop souvent appariés par leurs antécédents médicaux, la présence ou non d'un tabagisme, l'âge et le statut génital de la partenaire, la variabilité des périodes d'abstinence $[4,6,7,11,14,19,21]$.

\section{CONCLUSION}

Au total, il ressort de l'analyse des données disponibles, qu'il existe certainement une diminution progressive de la qualité spermatique liée au vieillissement, sans qu'un âge seuil puisse être individualisé. Les paramètres affectés sont le plus souvent le volume de l'éjaculât, la mobilité et la morphologie des spermatozoïdes. La numération semble plus modérément affectée. Des études comparatives supplémentaires, incluant un plus grand nombre de patients âgés avec des tranches d'âge clairement individualisées et des populations appariées pour les autres facteurs d'altération de la qualité spermatique, sont nécessaires. Du fait des grandes variations inter et intra individuelles des modifications spermatiques observées, les altérations spermatiques liées à l'âge sont quantitativement modérées. Il est probable que les conséquences sur la qualité spermatique des anomalies du système reproductif lié au vieillissement naturel des gonades et aux pathologies de la maturité seraient plus liées à l'âge dit physiologique du sujet qu'à son âge réel.

Les données publiées sur les caractéristiques spermatiques des hommes âgés ne permettent donc pas d'individualiser des critères pronostiques spécifiques de leur éventuelle fécondité en rapport avec leur âge. En d'autres termes, quelles que soient les caractéristiques spermatiques d'un sujet âgé désireux d'une éventuelle paternité, ceux-ci ne permettent pas de faire l'économie d'un bilan clinique et andrologique complet.

\section{REFERENCES}

1. AUGER J., KUNSTMANN J.M., CZYGLIK F., JOUANNET P. : Decline in semen quality among fertile men in Paris during the past 20 years. N. Engl. J. Med., 1995, $3:$ 281-285.

2. BICCHIERAY L., BEN FTIMA I., ALBERT M. et al. : Analyse morphometrique semi quantitative de l'histologie testiculaire au cours du vieillissement. Andrologie, 2003, $13: 288-297$

3. BREMNER W.J., VITIELLO M.V., PRINZ P.N. : Loss of circadian rythmicity in blood testosterone levels with aging in normal men. J. Clin. Endocrinol. Metab., 1983, 56 : 1278-1281.

4. CHECK J.H., SHANIS B., BOLLENDORF A. et al. : Semen characteristics and infertility in aging. Arch. Androl., 1989, 23 : 275-277.

5. ESKENASI B., WYROBEK A.J., SLOTER E. et al. : The association of age and semen quality in healthy men. Hum. Reprod., 2003, 18 : 447-454.

6. GALLARDO E., SIMON C., LEVY M. et al. : Effect of age on sperm fertility potential : oocyte donation as a model. Fertil. Steril., 1996, $66:$ 260-264.

7. HAIDL G., JUNG A., SCHILL W.B. : Aging and sperm function. Hum. Reprod., 1996, 11 : 558-560.

8. HERMANN M., UNTERGASSER G., RUMPOLD H. et al. : Aging of the male reproductive system. Exp. Gerontol., 2000, $35: 1267-1279$

9. JUNG A., SCHUPPE H.C., SCHILL. W.B. : Comparison of semen in older and younger men attending an andrology clinic. Andrologia, 2002, 34 : 116-122. 
10. KAISER F.E. : Sexuality in the elderly. Urol. Clin. North Am., 1996, $23: 99-109$.

11. KIDD S.A., ESKENAZI B., WYROBEK A.J. : Effects of male age on semen quality and fertility : a review of the literature. Fertil. Steril, 2001, $75: 237-248$.

12. MAHMOUD A.M., GOEMAERE S., EL-GAREM I. et al. : Testicular volume in relation to hormonal indices of gonadal function in community-dwelling elderly men. J. Clin. Endocrinol. Metab., 2003, $88:$ 179-184.

13. MEACHAM R.B., MURRAY M.J. : Reproductive function in the aging male. Urol. Clin. North. Am., 1994, 21 : 549-556.

14. MLADENOVIC I., MISIC S., PAPIC N. et al. : Sperm morphology and motility in different age populations. Arch. Androl., 1994, 32 : 197-205.

15. NEAVES W.B., JOHNSON L., PORTER J.C. et al. : Leydig cell numbers, daily sperm production, and serum gonadotropin levels in aging male. J. Clin. Endocrinol. Metab., 1984, 59 : 756-763.

16. NIESCHLAG E., LAMMERS U., FREISCHEM C.W. et al. : Reproductive functions in young fathers and grandfathers. $J$. Clin. Endocrinol. Metab., 1982, 55 : 676-681.

17. PAULSON R.J., MILLIGAN R.C., SOKOL R.Z. : The lack of influence of age on male fertility. Am. J. Obstet. Gynecol., 2001, $184: 818-824$.

18. PLAS E., BERGER P., HERMANN M., PFLUGER H. : Effects of aging on male fertility ? Exp. Gerontol., 2000, 35 : 543-551.

19. ROLF C., BEHRE H.M., NIESCHLAG E. : Reproductive parameters of older compared to younger men of infertiles couples. Int. J. Androl., 1996, $19: 135-142$.

20. SCHWARTZ D., MAYAUX M.J., SPIRA A et al. : Semen characteristics as a function of age in 833 fertile men. Fertil. Steril., 1983, $39: 530-535$.

21. SPANDORFER S.D., AVRECH O.M., COLOMBERO D. et al. : Effect of parental age on fertilization and pregnancy characteristics in couples treated by intracytoplasmic sperm injection. Hum. Reprod., 1998, 13 : 334-338.

22. VERMEULEN A., KAUFMAN J.M. Aging of the hypothalamopituitary-testicular axis in men. Horm. Res., 1995, 43 : 25-28.

23. VERMEULEN A., KAUFMAN J.M., GIAGULLI V.A. : Influence of some biological indices on sex hormone binding globulin and androgen levels in aging and obese males. J. Clin. Endocrinol. Metab., 1996, $81: 1821-1827$

Communication au $X X^{\circ}$ Congrès de la Société d'Andrologie de Langue Française, Orléans, 11-13 décembre 2003.

Manuscrit reçu : novembre 2003 ; accepté : janvier 2004.
ABSTRACT

Evolution of semen parameters with ageing

Louis SIBERT, Nathalie RIVES

The increasing life expectancy and the increasingly advanced age of parenthood due to socio-economic conditions in developed countries, combined with progress in medically-assisted procreation techniques account for the recent interest in the fertility of ageing men. Hormonal changes (primary testicular deficiency, decreased amplitude of hypothalamic GnRH secretion peaks), morphological and histological testicular changes (arteriolar sclerosis, Leydig cell and Sertoli cell degeneration, rarefaction of germ cells, thickening of the testicular tunica albuginea) related to physiological ageing are relatively well documented. The repercussions of these physiological changes on the quality of the semen of ageing men is difficult to clearly establish because of the limited data available and the marked interindividual variability of semen parameters. Globally, the quality of semen gradually decreases with age, although a cut-off age cannot be defined. The alterations observed essentially concern the volume of the ejaculate, and the mobility and morphology of spermatozoa. The sperm count appears to be less markedly affected. However, all of the other pathological and psychosocial factors frequently observed in ageing men must be taken into account in the interpretation of the data (changes of the bladder neck and genital tract, chronic diseases, drug treatments, smoking, decreased frequency of sexual intercourse, partner's age). Further comparative studies, including a larger number of elderly patients in clearly defined age-groups, with populations matched for other risk factors for alteration of semen quality, are necessary. Overall, a review of the literature does not reveal any specific prognostic criteria for age-related fertility. Regardless of the semen characteristics of an ageing man desiring possible paternity, a complete clinical and andrological assessment must be performed.

Key-Words: ageing male, fertility, semen volume, sperm count, motility, morphology 